
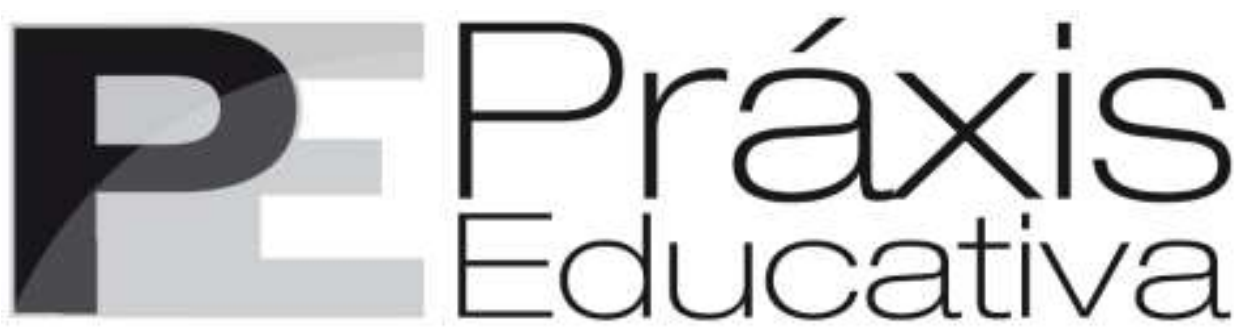

ISSN 1809-4031

elSSN 1809-4309

https://doi.org/10.5212/PraxEduc.v.17.19696.001

Seção: Tradução

\title{
A política da sociologia crítica das políticas: mobilidades, amarras e redes
} de elite*

\section{The politics of critical policy sociology: mobilities, moorings and elite} networks

\section{La política de la sociología crítica de las políticas: movilidades, amarres y redes de élites}

Glenn C. Savage*

(D) https://orcid.org/0000-0001-6495-6798

Jessica Gerrard ${ }^{* * *}$

(D) https://orcid.org/0000-0001-9011-6055

Trevor Gale ${ }^{* * * *}$

(D) https://orcid.org/0000-0003-3927-9267

Tebeje Molla ${ }^{* * * * *}$

(D) https://orcid.org/0000-0002-6848-3091

Resumo: Este artigo reflete sobre o que significa fazer sociologia crítica das políticas na mudança de contextos teóricos, empíricos e metodológicos da educação. Concentramos nossas lentes analíticas em duas considerações principais. Em primeiro lugar, refletimos sobre a política da criticidade, examinando diferentes afirmações e debates sobre o que significa fazer pesquisa crítica e ser um pesquisador crítico da política educacional, prestando atenção especial a como os sociólogos das políticas posicionam seu trabalho em relação ao poder da elite e às redes de políticas. Em segundo lugar, apoiamo-nos sobre essas bases para considerar a tendência de pesquisar mobilidades dentro da sociologia crítica das políticas, de modo a argumentar que a pesquisa "siga a política" contemporânea corre o risco de orientar os pesquisadores para

\footnotetext{
* Tradução de Janete Bridon. Revisão técnica de Jefferson Mainardes (UEPG).

${ }^{* *}$ Professor Associado da University of Western Australia na School of Social Sciences e na Graduate School of Education. E-mail: <glenn.savage@uwa.edu.au>.

*** Professora da University of Melbourne (Austrália). E-mail: <jessica.gerrard@unimelb.edu.au>.

**** Professor da University of Glasgow (Escócia). E-mail: <Trevor.Gale@glasgow.ac.uk>.

***** Professor da Deakin University (Austrália). E-mail: <t.mekonnen@deakin.edu.au>.
} 
A política da sociologia crítica das políticas: mobilidades, amarras e redes de elite

os problemas e as agendas já estabelecidas por agentes políticos de elite e organizações, enquanto obscurece as forças não tão móveis que continuam a definir as políticas e as práticas educacionais. Também levantamos questões sobre as redes de elite e os níveis privilegiados de recursos normalmente necessários para conduzir esse tipo de pesquisa. Em conclusão, convidamos a uma discussão mais aprofundada sobre a política de produção de conhecimento e os desafios para os sociólogos das políticas que buscam ser críticos em contextos de mudança.

Palavras-chave: Sociologia da política. Criticidade. Mobilidade. Elites.

\begin{abstract}
This article reflects on what doing critical policy sociology means in shifting theoretical, empirical and methodological contexts of education. We focus our analytical lens on two primary considerations. First, we reflect on the politics of criticality, examining differing claims and debates about what it means to do critical research and be a critical researcher of education policy, paying particular attention to how critical policy sociologists position their work in relation to elite power and policy networks. Second, we build on these foundations to consider the trend towards researching mobilities within critical policy sociology, arguing that contemporary 'follow the policy' research risks orienting researchers to the problems and agendas already established by elite policy agents and organisations, while obscuring the not-so-mobile forces that continue to define education policy and practice. We also raise questions about the elite networks and privileged levels of resourcing typically required to conduct this kind of research. In conclusion, we invite further discussion on the politics of knowledge production and challenges for policy sociologists seeking to be critical in shifting contexts.
\end{abstract}

Keywords: Policy Sociology. Criticality. Mobility. Elites.

Resumen: Este artículo reflexiona sobre lo que significa hacer sociología crítica de las políticas en los cambiantes contextos teóricos, empíricos y metodológicos de la educación. Centramos nuestras lentes analíticas en dos consideraciones principales. Primero, reflexionamos sobre la política de la criticidad, examinando diferentes afirmaciones y debates sobre lo que significa hacer investigación crítica y ser un investigador crítico de la política educativa, prestando especial atención a cómo los sociólogos políticos posicionan su trabajo en relación con el poder de la élite y las redes de políticas. En segundo lugar, nos basamos en estas bases para considerar la tendencia a las movilidades de investigación dentro de la sociología crítica de la política, con el fin de argumentar que la investigación contemporánea de "seguimiento de políticas" corre el riesgo de orientar a los investigadores hacia problemas y agendas ya establecidas por actores políticos de élite y organizaciones, al tiempo que oscurece las fuerzas no tan móviles que continúan definiendo las políticas y prácticas educativas. También planteamos preguntas sobre las redes de élite y los niveles privilegiados de recursos que normalmente se requieren para realizar este tipo de investigación. En conclusión, invitamos a una mayor discusión sobre la política de producción de conocimiento y los desafíos para los sociólogos de políticas que buscan ser críticos en contextos cambiantes.

Palabras clave: Sociología de la política. Criticidad. Movilidad. Élites.

\title{
Introducão
}

Neste artigo, refletimos sobre o que significa ser um "sociólogo crítico das políticas" na mudança de contextos teóricos, empíricos e metodológicos da educação. O que significa fazer pesquisa "crítica" em sociologia das políticas? O que significa "ser crítico", engajar-se na "crítica" ou adotar uma "disposição crítica"? Quais são algumas das tensões e dos desafios para aqueles que buscam se engajar em análises sociológicas "críticas" da política educacional? Dado que qualquer tentativa de mapear de forma abrangente o campo da sociologia crítica das políticas para fornecer uma resposta a tais questões vai além do escopo de um único artigo, focamos nossas lentes analíticas em duas considerações principais, que estão intimamente relacionadas e acreditamos que são frutíferas para provocar uma discussão sobre a evolução do trabalho dos sociólogos críticos das políticas. Em primeiro lugar, refletimos sobre a politica da criticidade (politics of criticality), no sentido de examinar diferentes afirmações e debates sobre o que significa fazer pesquisa crítica e ser um

Práxis Educativa, Ponta Grossa, v. 17, e2219696, p. 1-18, 2022 Disponível em: <https://revistas2.uepg.br/index.php/praxiseducativa $>$ 
pesquisador crítico de políticas educacionais. ${ }^{1}$ Ao refletirmos sobre algumas das principais tendências históricas e emergentes do campo, incluindo nossas próprias histórias e tentativas de moldar a pesquisa da sociologia crítica das políticas, consideramos diferentes modos de crítica e prestamos atenção especial a como os sociólogos das políticas posicionam seu trabalho em relação ao poder da elite e às redes das políticas. Em segundo lugar, para ilustrar como a política da criticidade está evoluindo, consideramos as tendências para a pesquisa de "mobilidades das políticas" dentro da sociologia crítica das políticas. Focamos especialmente nos apelos para o engajamento em pesquisas que "seguem as políticas", argumentando que, embora essa forma de pesquisa fale de uma necessidade crucial de examinar o poder e o impacto das redes globais de elite, corre o risco de orientar os pesquisadores para problemas e agendas já estabelecidas pelos agentes elitistas das políticas e organizações, enquanto obscurece as forças não tão móveis que continuam a definir as políticas e as práticas educacionais. O desejo de seguir os fluxos de políticas globais também levanta questões sobre as redes das elites e os níveis privilegiados de recursos normalmente necessários para conduzir tais pesquisas. Em conclusão, convidamos a uma discussão mais aprofundada sobre a política de produção de conhecimento e os desafios para os sociólogos políticos que buscam ser críticos em contextos de mudança. Argumentamos que há uma necessidade contínua de criticar o crítico e questionar se os pesquisadores que buscam "criticar" as redes das elites podem, em vez disso, estar participando da pesquisa das elites, pelas elites e para as elites.

\section{A política da criticidade}

A história da sociologia crítica das políticas educacionais foi infundida com afirmações e debates diferentes e, muitas vezes, concorrentes sobre o que significa fazer pesquisa crítica (REGMI, 2019) e, como autores deste artigo, contribuímos de diferentes maneiras para o desenvolvimento histórico desses debates (veja a seguir). Essas diferentes posições falam sobre a política da criticidade inerente e levantam questões sobre os vários papéis que os pesquisadores críticos desejam desempenhar. Ao refletirmos sobre a política da criticidade, afirmamos, desde o início, que a grande maioria dos pesquisadores que associam seus estudos à sociologia crítica das políticas implícita ou explicitamente refletem um compromisso com uma política de pesquisa que busca desafiar as estruturas do poder dominantes e acordos políticos e agenciamento de políticas. Em alguns casos, esses compromissos políticos se estendem ao desenvolvimento de pesquisas que procuram explicitamente inspirar mudanças sociais, políticas ou educacionais. Por exemplo, se considerarmos os estudos educacionais de base na sociologia crítica das políticas, é claro que a abordagem tem sido caracterizada por objetivos políticos e emancipatórios normativos, com estudiosos argumentando que uma forma de crítica que busca formular visões de um futuro melhor é central para fazer pesquisas críticas.

O enquadramento de sociologia crítica das políticas educacionais de Gale (2001, p. 379), por exemplo, descreve-a como centrada na "crítica das práticas sociais opressivas" e ancorada em compromissos políticos e morais para melhorar o mundo. Ao conectar a análise das políticas educacionais ao interesse sociológico nas relações entre "problemas pessoais" e "questões públicas" (MILLS, 1959, p. 8), Gale argumenta que a sociologia crítica das políticas fornece um meio para intervir nas políticas educacionais para uma mudança positiva; ou, como coloca o estudioso político Prunty (1985, p. 136), gera pesquisa, “[...] ancorada na visão de uma ordem moral

\footnotetext{
1 Reiteramos que nosso propósito não é "mapear" de forma abrangente o campo diverso da sociologia crítica das políticas educacionais, nem historicamente, nem em termos de principais autores. Em vez disso, oferecemos algumas reflexões concretas sobre a "política da criticidade", a fim de fornecermos bases para nossa análise nas seções a seguir. Também é importante notarmos que nossas reflexões estão focadas principalmente na linha distinta britânica/australiana da sociologia das políticas que surgiu a partir dos anos de 1980 (ver a análise sobre esse estudo em OZGA, 2019).
}

Práxis Educativa, Ponta Grossa, v. 17, e2219696, p. 1-17, 2022 Disponível em: < https://revistas2.uepg.br/index.php/praxiseducativa $>$ 
em que a justiça, a igualdade e a liberdade individual não são comprometidas pela avareza de alguns". Blackmore (2014, p. 515), da mesma forma, argumenta que "fazer a diferença" é central para a criticidade, que "[...] requer a negociação de tensões entre o particular/universal, trabalhar com e sobre as ortodoxias dominantes, enquanto fornece maneiras alternativas de fazer e ver o mundo, sobre trabalhar dentro e nas regras do jogo". No relato de Rizvi e Lingard (2010, p. 51), a sociologia crítica das políticas é similarmente descrita como impulsionada por propósitos "normativos e imaginativos" que "[...] devem não apenas descrever as relações de poder e os processos por meio dos quais as políticas são desenvolvidas e alocadas, mas também deve apontar para estratégias para uma mudança progressiva que pode desafiar as estruturas e as práticas opressivas". Ao apresentarem esses argumentos, tais estudiosos refletem uma linhagem da teoria crítica associada à Escola de Frankfurt, que visa ir além de simplesmente compreender e criticar as relações sociais, políticas e econômicas, para buscar mudar o mundo (SCOT'T, 1978). Na verdade, Horkheimer (1972) argumentou que a teoria só era crítica se fosse além de explicar o que havia de errado com o mundo para desenvolver uma visão normativa para a transformação.

No entanto, agendas políticas explícitas e emancipatórias nem sempre estão presentes, nem são preferidas, na pesquisa de sociologia crítica das políticas educacionais. Como Sellar, Savage e Gorur (2014) argumentam, existem diferentes maneiras de compreender o que significa "ser crítico", e muitos estudiosos da sociologia crítica das políticas se engajam em um tipo diferente de política (politics) que não busca articular explicitamente soluções ou visões de um futuro desejado. Essa forma de pesquisa crítica frequentemente envolve estratégias como mapear e desempacotar relações e redes de poder, examinar como as formações políticas e sociais são montadas e criticar a construção de soluções/problemas das políticas. Tal pesquisa normalmente examina, critica e procura explicar os fenômenos sociais, mas sem estender isso a uma visão específica para a mudança que outros veem como parte integrante do estudo crítico. Seguindo Latour (2004), podemos dizer que tais estudiosos sinalizam um modo alternativo de crítica e um tipo diferente de disposição crítica, que busca reunir ideias de maneiras que ofereçam aos leitores "arenas" nas quais se engajem em conversas críticas. Isso não significa que tal pesquisa não possa implicar um futuro alternativo preferido ou um conjunto de arranjos por meio do engajamento na crítica, mas que não são feitas tentativas para delinear explicitamente quais seriam essas alternativas. Essa abordagem é comum na teoria ator-rede (actor-network theory - ANT). Fenwick e Edwards (2010) argumentam, por exemplo, que a teoria ator-rede busca "intervir" em formas estabelecidas de pensamento, em vez de dizer aos leitores "o que pensar".

Uma posição amplamente semelhante pode ser vista em estudos que se baseiam na filosofia e na teoria pós-estruturalista, em que os pesquisadores, muitas vezes, colocam em primeiro plano os benefícios da crítica e das formas de problematização, em vez de articular soluções explícitas ou visões para a mudança. Aqui, o ato de mapear, no qual as construções discursivas, materiais e afetivas das políticas são "desnudadas", é frequentemente entendido como uma intenção e um impacto potencial da pesquisa. Crítica e mudança são vistas como intimamente conectadas (BACCHI, 2000). Com base em Gilles Deleuze, por exemplo, Thompson (2019, p. 45) argumenta que "[...] o valor em teoria está na articulação de questões ou problemas, em vez de soluções", acrescentando que a crítica dos problemas nos permite melhor "compreender a constituição de nossa condição" (THOMPSON, 2019, p. 46). Gerar soluções, ele argumenta, é circunscrito por sua relação limitada com problemas predefinidos, enquanto a problematização "[...] nos força a um encontro em que algo novo emerge, novos pensamentos, novas possibilidades, novas compreensões" (THOMPSON, 2019, p. 46).

Para muitos, deixar de articular fins normativos baseados em soluções é proposital. Rasmussen (2016), por exemplo, sugere que uma lente emancipatória voltada a fornecer soluções pode, muitas vezes, presumir sua capacidade de sair das tecnologias dominantes de governança que

Práxis Educativa, Ponta Grossa, v. 17, e2219696, p. 1-18, 2022 Disponível em: < https:// revistas2.uepg.br/index.php/praxiseducativa $>$ 
contornam as vidas dos participantes da pesquisa e do trabalho acadêmico para determinar o que é "bom" e "justo". Baseando-se em Berlant (2011), Rasmussen (2016) sugere que a postura potencialmente não reflexiva sobre a qual as agendas emancipatórias podem apoiar-se pode servir como uma espécie de "otimismo cruel". Claro, embora concordemos que o trabalho dos pesquisadores é limitado, também é importante questionarmos o quão prevalente é essa tendência para a não-reflexividade entre os sociólogos críticos das políticas. $\mathrm{Na}$ verdade, com base nas articulações de Bourdieu da sociologia reflexiva (BOURDIEU; WACQUANT, 1992), enfatizamos que um compromisso com a autorreflexividade crítica é central para a pesquisa crítica (ver KENWAY; MCLEOD, 2004) e questionar se a pesquisa que carece de tal reflexividade pode ser considerada "crítica" de alguma forma.

Embora existam orientações distintas para a criticidade, é importante reconhecermos que existem alguns pontos fortes em comum que unem os pesquisadores da educação sob o guardachuva da sociologia crítica das políticas. Esse é especialmente o caso em termos de como os pesquisadores entendem as relações entre política (politics), criticidade e futuro. Por exemplo, quer os pesquisadores articulem ou não soluções ou agendas políticas explícitas para a mudança, vemos as diversas formas de estudos associadas à sociologia crítica das políticas como sustentadas por formas preferidas de política (politics) que, muitas vezes, apontam para possibilidades futuras. Em outras palavras, mesmo quando os estudiosos procuram evitar a formulação explícita de soluções ou de agendas futuras, suas análises não refletem menos os posicionamentos ou os objetivos políticos, mesmo que estes possam ser implícitos e não declarados. Assim, embora concordemos com Thompson (2019) de que o ato de buscar soluções pode ser circunscrito por sua relação com um problema, vemos o próprio ato de escolher em qual arena intervir e problematizar, e os recursos intelectuais utilizados nos processos de intervenção e de problematização, também politicamente circunscritos. Na verdade, a escolha de desempacotar, problematizar ou reunir ideias para uma discussão crítica em torno de um objeto de investigação é claramente uma escolha restrita que carrega consigo uma política preferida, independentemente de os pesquisadores serem explicitamente autorreflexivos sobre isso ou não. Os processos de construção de soluções são tão limitados quanto os atos de problematização e igualmente capazes de produzir novas possibilidades de pensar e compreender o mundo.

Além disso, a escolha de não articular soluções explicitamente também carrega uma forma preferida de política: isto é, o que não fazemos é um ato político. Não há possibilidade para os acadêmicos contornar ou "sentar-se fora" das condições políticas de possibilidade em que seu trabalho está inserido e, embora o envolvimento na autorreflexividade seja integral, não fornece um ponto de vista externo que possa libertar alguém de sua inevitável limitação e parcialidade política. Argumentos em estudos feministas, queer, antirracistas e pós-coloniais fornecem alguns pontos de vista a partir dos quais essas dinâmicas são abordadas. Para muitos pesquisadores críticos nesses campos, é impossível escapar de "ser político" ou ser posicionado como tendo interesses adquiridos, porque a posicionalidade de suas pesquisas já a anuncia como politicamente enredada e como articuladora de relações particulares entre passado/presente/futuro. Tomemos, por exemplo, a obra Decolonizing methodologies: research and indigenous peoples, de Smith (1999) ${ }^{2}$, que nos lembra que a política de pesquisa está profundamente conectada à relação de um pesquisador com ela. Para Smith, isso envolve ver o próprio significado de "pesquisa" como "[...] inextricavelmente ligada ao imperialismo europeu e ao colonialismo" (SMITH, 1999, p. 1). Em nossa visão, o ato inevitavelmente político de pesquisa significa que é problemático ver a problematização como uma forma preferível de crítica à construção de soluções. Em vez disso, a problematização deve ser vista

\footnotetext{
${ }^{2}$ Nota de tradução: Publicada no Brasil com o título “Descolonizando metodologias: pesquisa e povos indígenas”, pela editora da Universidade Federal do Paraná (UFPR).
} 
como parte integrante da formulação crítica de soluções para aqueles que optam por engajar-se em tal trabalho, e a formulação de soluções não deve ser vista como necessariamente não crítica.

Outro ponto poderoso condutor da pesquisa da sociologia crítica das políticas educacionais, que é de interesse central para este artigo, é uma orientação crítica em relação aos atores, às redes e ao poder da elite. Sociólogos críticos das políticas tipicamente enquadram seus estudos, implícita ou explicitamente, como um desafio às formas dominantes e elitistas de poder político, social, econômico e cultural. Essa orientação é antiga na sociologia da educação (por exemplo, YOUNG, 1971) e reflete tendências históricas mais amplas na sociologia que Mike Savage e colaboradores analisaram nas últimas décadas (ver SAVAGE, 2015; SAVAGE; WILLIAMS, 2008). A sociologia crítica das políticas emergiu historicamente de desenvolvimentos na sociologia da educação, com evoluções significativas ocorrendo desde o final dos anos de 1980 até o início dos anos 2000, à medida que um grupo conectado de estudiosos buscava adotar linhas críticas de pesquisa em reação a contextos políticos em mudança e a transformações associadas no trabalho acadêmico (OZGA, 2019). No centro deste trabalho de base, estava o desejo de criticar e desafiar a evolução dos arranjos das políticas e da governança associados à ascensão do neoliberalismo, especialmente no Reino Unido, sob um governo conservador (BALL, 1997), e, também, na Austrália, sob um governo trabalhista (LINGARD, 1993). Apesar de distintas, várias tensões de sociologia crítica na educação evoluíram nos Estados Unidos durante o mesmo período, em resposta ao reaganismo e aos desenvolvimentos subsequentes (APPLE, 1996), embora nem sempre com o forte foco das "políticas" que animou o surgimento do campo dentro e em todo o Reino Unido e na Austrália.

Com o passar do tempo, mercantilização, corporatização, novos modos de responsabilização (accountability), cultura de auditoria, escolha de escola, devolution ${ }^{3}$ e outros fenômenos tipicamente associados a formas neoliberais de governança na educação têm sido áreas maduras de crítica para sociólogos críticos das políticas. Aqui, muitos dos principais dilemas sociológicos relativos à lógica do poder (por exemplo, em relação à economia, ao gênero, à raça, à sexualidade, à habilidade e outros), bem como as interseções entre essas forças, têm sido preocupações centrais na análise e na compreensão das políticas educacionais e da política (politics) (ver DAVID; WEINER; ARNOT, 2000; GILLBORN, 2005; GUNTER, 2018; HOGARTH, 2018). Nas últimas décadas, essas áreas de foco evoluíram e se expandiram para prestar atenção mais explícita à globalização e ao aumento e impacto associados de novas "mobilidades das políticas", com um interesse particular em examinar e criticar as redes globais da elite de atores e de organizações que agora exercem influência significativa sobre a formação de ideias e de práticas de políticas educacionais dentro e entre as nações (ver GULSON et al., 2017; BALL, 2016; RIZVI; LINGARD, 2010). Novamente, essa mudança reflete tendências mais amplas na sociologia, por meio das quais os estudiosos da mobilidade têm buscado compreender o papel das elites transnacionais por meio de um foco conceitual nas formas mutantes e nas forças da globalização (CASTELLS, 1996). É essa tendência ainda emergente de pesquisar mobilidades das políticas na pesquisa de políticas educacionais para a qual, agora, voltamos nossa atenção, a fim de ilustrarmos as implicações dessa mudança de olhar político na pesquisa da sociologia crítica das políticas.

\section{Pesquisa de mobilidades e novas redes globais de preocupação}

As mudanças nas condições da globalização e a ampla "virada das mobilidades" nas Ciências Sociais (URRY, 2003) remodelaram, significativamente, as teorias, os métodos e os focos

\footnotetext{
${ }^{3}$ Nota de revisão: Devolution refere-se à distribuição de poder e de financiamento entre o governo e as escolas, bem como mudanças na governança, gestão e financiamento das escolas. Envolve decisões de gestão baseadas na escola, autonomia da escola, autogestão, autonomia e gestão descentralizada.
}

Práxis Educativa, Ponta Grossa, v. 17, e2219696, p. 1-18, 2022 Disponível em: < https:// revistas2.uepg.br/index.php/praxiseducativa $>$ 
empíricos da sociologia crítica das políticas nas últimas décadas. Na verdade, a sociologia crítica das políticas educacionais toma, cada vez mais, como ponto de partida, a realidade empírica da "mobilidade" e se concentra no desenvolvimento de novas ferramentas analíticas para compreendê-la e abordá-la. De particular relevância para os sociólogos críticos das políticas são os fluxos globais novos e intensificados de ideias, de práticas, de atores, de tecnologias e de capital de políticas, possibilitados pelo avanço tecnológico e pela conectividade do capitalismo avançado (GULSON et al., 2017). Essas condições têm repercussões espaciais e temporais importantes. Espacialmente, por exemplo, a necessidade de ir além do "nacionalismo metodológico", que trata o estado-nação como um sistema limitado e como um contêiner analítico a priori (BECK, 2007), teve uma aceitação considerável. Como Gulson et al. (2017, p. 224) argumentam, "[...] a presença de novas redes de políticas e relacionalidades significa que a formulação de políticas educacionais e governança não estão mais ocorrendo simplesmente dentro dos limites prefigurados do estadonação"4. Temporalmente, pesquisas recentes prestaram atenção especial à velocidade da conectividade como uma condição de mobilidade que molda a forma como as políticas "viajam" e são atuadas (enacted) (LEWIS; HOGAN, 2019). Como observam Peck e Theodore (2015), a questão não é simplesmente que tudo se tornou "rápido", mas que a velocidade e a mobilidade são coconstituídas com as práticas políticas, presumindo e possibilitando a velocidade, na medida em que é central para os significados e as práticas de produção das políticas e sua atuação (enactment).

Pensar de forma diferente sobre o espaço e o tempo tem levado a novas conceitualizações da natureza das conexões e dos fluxos globais, evidentes, por exemplo, em trabalhos recentes que buscaram entender as mobilidades por meio de lentes topológicas. Relatos topológicos " [...] procuram interromper e re-renderizar narrativas dominantes sobre escala, relações locais-globais, o exercício de poder e outras dimensões centrais para teorizar as novas espacialidades da globalização" (SAVAGE, 2020, p. 327). Ao rejeitar a noção euclidiana de espaço como um conjunto de "coordenadas fixas" a priori sobre as quais as relações políticas, das políticas e de poder atuam, relatos topológicos abordam como novos espaços relacionais trazem o próximo e o distante em tessituras que não podem ser entendidas em termos de noções de escala territorial (HARTONG; PIATTOEVA, 2019).

$\mathrm{Na}$ tentativa de forjar novos entendimentos espaciais e temporais, os sociólogos críticos das políticas educacionais têm se apropriado de uma ampla gama de recursos teóricos e disciplinares para abordar a dinâmica da globalização. Especialmente proeminentes foram os insights da geografia crítica (GULSON et al., 2017), da teoria do agenciamento (SAVAGE, 2020) e da análise de rede de políticas (BALL, 2016). Embora essas tendências não possam ser eliminadas como uma orientação singular, há convergências claras, principalmente em sua atenção às relações móveis. Novamente, tais pesquisas agora tendem a tratar a mobilidade e o movimento como pontos de entrada conceituais e metodológicos, a partir dos quais conexões e acontecimentos contemporâneos são traçados e criticados, enquanto, ao mesmo tempo, não tomam categorias sociológicas tradicionais - como nação, estado, classe, raça ou gênero - como dadas ou assumem a natureza e a forma de sua existência (ver GORUR et al., 2019). Da mesma forma, racionalidades e práticas de governança mais amplas (por exemplo, "neoliberalismo") são entendidas não como predeterminadas, mas como devires dinâmicos, diversamente traduzidos, alterados e agenciados de diferentes maneiras ao longo do tempo e do espaço. O neoliberalismo pode ser entendido, nesse sentido, como uma "tecnologia móvel" (ONG, 2007) que se manifesta de formas dependentes do contexto.

Embora a pesquisa relacionada às mobilidades leve o campo a novas direções, ela também mantém o foco crítico nos atores, nas redes e no poder de elite que, historicamente, definiu a

\footnotetext{
${ }^{4}$ Nós adicionaríamos a isso o "subnacional", especialmente em pesquisas focadas em sistemas federais.
}

Práxis Educativa, Ponta Grossa, v. 17, e2219696, p. 1-17, 2022 Disponível em: <https:// revistas2.uepg.br/index.php/praxiseducativa> 
sociologia das políticas educacionais. Assim, enquanto a lente analítica está cada vez mais focada na mudança dos fluxos globais e topológicos de ideias, atores e práticas de políticas, a maior parte dos sociólogos críticos das políticas continuam a enquadrar seus estudos, implícita ou explicitamente, falando para e/ou contra atores, redes e poder de elite. Por exemplo, um argumento comum em estudos recentes de sociologia das políticas, incluindo o trabalho do primeiro autor deste artigo, é que as interações mutantes entre governos, think tanks, filantropias, organizações supranacionais, empreendedores de políticas e outros atores estão resultando em redes globais complexas que estão redistribuindo o poder e a influência entre os espaços políticos de novas maneiras, remodelando as formas de evidência, expertise e influência que moldam as políticas educacionais (BALL, 2016; HOGAN, 2016; SAVAGE, 2016). O objetivo principal desta pesquisa é mapear, compreender e avaliar criticamente o trabalho das "elites políticas" globais que ocupam posições de poder e de influência que permitem que esses atores exerçam uma influência significativa sobre as reformas e os processos de políticas educacionais. ${ }^{5}$

Vemos esta pesquisa como uma intervenção necessária nas redes mutantes de poder que orientam o desenvolvimento e a atuação (enactment) de políticas educacionais. No entanto, também vemos a necessidade de considerar mais criticamente o posicionamento do "pesquisador crítico" nesse corpo de pesquisa em evolução. Se trabalharmos a partir da premissa de que os pesquisadores e os "sujeitos" de sua investigação são mutuamente construídos pelas criações teóricas, conceituais e metodológicas de seu trabalho, então não devemos apenas perguntar como a pesquisa de mobilidades está remodelando o tipo de pesquisa que conduzimos, mas também como essa pesquisa está reconstituindo o próprio trabalho acadêmico. Essa crítica da crítica é necessária porque estamos preocupados com o fato de que, em alguns casos, a pesquisa sobre os fluxos globais de políticas necessita recompensas e produz um subconjunto altamente privilegiado de pesquisadores globalmente móveis, que, muitas vezes, são compelidos a se tornarem integrados às próprias redes de políticas da elite que eles procuram compreender e criticar (ver também METCALFE, 2017). Esse é especialmente o caso quando os pesquisadores de políticas educacionais buscam envolver-se nos tipos de projetos de pesquisa que "seguem as políticas" defendidos por geógrafos críticos como Peck e Theodore (2012, 2015), que sugerem que rastrear e criticar o movimento global das políticas de maneira ideal requer que os pesquisadores se envolvam em um empreendimento etnográfico altamente móvel voltado a acompanhar os principais atores e locais envolvidos na produção das políticas. Embora reconheçamos que nem toda sociologia crítica das políticas educacionais está preocupada em seguir fisicamente as redes globais de elite, e algumas formas de pesquisas que "seguem a política" se centram em pesquisa online (rastrear redes, analisar políticas e participar de entrevistas por meio de pesquisa "desktop"), é justo dizermos que o campo foi fortemente caracterizado por um interesse em adotar essa abordagem etnográfica móvel (LEWIS, 2020).

Em nossa visão, as formas de mobilidade exigidas dos pesquisadores para se engajarem nos esforços etnográficos móveis, promovidos por muitos pesquisadores que "seguem as políticas", baseiam-se nas relações com o poder e a influência da elite que precisa ser mais bem explicada e sustentada para a crítica. Por exemplo, os pesquisadores, muitas vezes, encontram-se "pesquisando" à medida que seguem os processos das políticas, viajando pelo mundo e entrevistando agentes das políticas altamente influentes e da elite (ver NEAL; MCLAUGHLIN, 2009). Essas condições experienciais de pesquisa sobre mobilidade de políticas se apoiam e criam formas de trabalho de pesquisa acadêmica, nas quais o trabalho em rede com redes de elite se torna

\footnotetext{
${ }^{5}$ Embora o foco desta pesquisa esteja nas elites que são capazes de exercer especificamente influência significativa sobre a formulação das políticas educacionais, reconhecemos que, na teoria política e sociológica mais ampla, o termo "elite" assume muitas formas (por exemplo, elite econômica, elite cultural, elite política, elite do poder e outras) e, também, é altamente contestada (ver, por exemplo, MILNER, 2015).
}

Práxis Educativa, Ponta Grossa, v. 17, e2219696, p. 1-18, 2022 Disponível em: <https://revistas2.uepg.br/index.php/praxiseducativa $>$ 
central. Com base em Ozga (2011), há, portanto, um tipo particular de habitus e inter-relação de elite formado entre pesquisador e agente da política por meio de tais interações e do conhecimento que produz. Como acontece com todas as pesquisas humanas, essas são interações interpessoais complexas que envolvem uma "performance" profundamente incorporada de um pesquisador interessado e experiente, que é capaz e se sente confortável em contextos de elite. Isso se estende além da inter-relação que ocorre em uma entrevista específica com as redes que são necessárias e cultivadas para permitir que alguém mapeie e rastreie os agentes das políticas globais influentes. Ser um pesquisador que "segue a política", por conseguinte, não só requer que alguém execute um "habitus de elite", mas também se baseie em privilégios de elite e formas de acesso que permitam aos pesquisadores, em primeiro lugar, realizar tais pesquisas. Globalmente, por exemplo, poucos podem ter o privilégio de viajar pelo mundo para pesquisar a elite mundial. ${ }^{6}$ Isso levanta questões desafiadoras sobre a posição inserida do pesquisador nas próprias redes de elite que procuram criticar. O que acontece, portanto, quando os pesquisadores (e o conhecimento que eles produzem) ficam inseridos nos fluxos de mobilidade que procuram pesquisar? Até que ponto os pesquisadores podem se inserir nas próprias redes de elite que procuram examinar criticamente? Há, consequentemente, um risco real de que os pesquisadores que buscam "criticar" as redes de elite possam, em vez disso, estar participando do que podemos chamar de pesquisa de elites, pelas elites e para as elites. ${ }^{7}$

Existem também importantes dimensões de gênero e racialidade que precisam ser mais bem reconhecidas. Por exemplo, mulheres pesquisadoras de políticas refletiram sobre como seu gênero pode contribuir para ser percebido como não ameaçador e inexperiente (DUKE, 2002; OZGA; GEWIRTZ, 1994). Além do campo da sociologia das políticas, as reflexões de Maylor (2009) sobre a experiência de pesquisa das mulheres negras destacam os impactos do racismo na academia e no contexto da própria pesquisa. Embora não aborde os contextos políticos diretamente, Mirza (2006 apud MAYLOR, 2009, p. 60) demonstra como “[...] ser um corpo 'fora do lugar' nas instituições Brancas tem custos emocionais e psicológicos para o portador dessa diferença". Ao escreverem sobre o contexto australiano, Thunig e Jones (2020) chamam atenção para as maneiras pelas quais os pesquisadores indígenas, de longe uma minoria dentro da academia, devem gerenciar as expectativas institucionais e colegiais de fazer o "trabalho duro" de desenvolvimento e atuação (enactment) de políticas indígenas dentro de suas instituições, limitando suas capacidades para construir suas prioridades de pesquisa. Essas reflexões mais amplas sobre as experiências de marginalização, que podem tornar alguns pesquisadores visíveis de algumas maneiras, mas invisíveis de outras, apontam para a profunda importância de como os pesquisadores são posicionados, compreendidos e julgados dentro da pesquisa baseada em políticas e na academia de forma mais ampla. Â luz dessas preocupações, acreditamos que há uma necessidade de perguntar até que ponto os pesquisadores têm as oportunidades e as redes para envolver-se em uma pesquisa que "siga a política" e as barreiras que podem existir para envolver-se nesse estudo. Queremos enfatizar que não é apenas o "quem" e o "como" da formulação de políticas que importa (GALE,

\footnotetext{
${ }^{6}$ Reconhecemos que nem todos os acadêmicos estão colocados da mesma forma. No entanto, argumentamos que qualquer pesquisador (de um estudante de Pós-Graduação a um professor bem pago), que tem acesso aos fundos necessários para viajar globalmente e pesquisar elites globais, ocupa uma posição altamente privilegiada, especialmente quando entendida em relação a um contexto mais amplo de global desigualdade social e econômica.

7 Percebemos que nosso argumento, aqui, gira em torno de uma questão sobre se os acadêmicos devem ser considerados atores de elite ou não, e convidamos a uma discussão mais aprofundada sobre isso. É interessante notarmos que Scott (2008) define elites não apenas em termos de pessoas com altas rendas, mas aquelas que são capazes de exercer formas significativas de poder. Em nossa opinião, muitos acadêmicos representam o que Scott (2008, p. 33) chama de "elite de especialistas", isto é, aqueles que são capazes de exercer o poder por meio do uso de "corpos especializados de conhecimento técnico". Claro, reconhecemos que, dentro dessa categoria, o poder de influenciar, por meio do conhecimento, é distribuído de forma desigual entre os acadêmicos.
}

Práxis Educativa, Ponta Grossa, v. 17, e2219696, p. 1-17, 2022 Disponível em: <https://revistas2.uepg.br/index.php/praxiseducativa $>$ 
A política da sociologia crítica das políticas: mobilidades, amarras e redes de elite

2003), mas também o "quem" do pesquisador. Perguntamos, portanto, "quem” é mais provável e capaz de ser um pesquisador que "segue a política" e quem não é? E por quê?

\section{Problemas de elite e agendas de pesquisa}

É igualmente importante refletirmos criticamente sobre como uma abordagem de mobilidades de política pode orientar os pesquisadores para os problemas e as agendas já estabelecidas pelos agentes de elite das políticas e das organizações, independentemente de os pesquisadores buscarem engajar-se em "seguir a política" ou não. Em outras palavras, estamos interessados em que tipos de problemas políticos, agendas e áreas de enfoque empírico entram na óptica para exame, como parte de um compromisso de pesquisar mobilidades dentro da sociologia crítica das políticas, e o que é silenciado ou deixado de fora. Por exemplo, embora o domínio absoluto de pesquisas recentes sobre o papel da Organização para a Cooperação e Desenvolvimento Econômico (OCDE) - e as principais tecnologias de políticas que ela produziu, como o Programa Internacional de Avaliação de Estudantes (Pisa) - pode ser parcialmente considerado como uma resposta necessária às mudanças que impulsionam a reforma educacional global, podemos também dizer que o crescente corpo de pesquisas sobre a OCDE corre o risco de reificar a organização e seu domínio. A OCDE apresentou argumentos poderosos de que os sistemas educacionais podem ser entendidos por meio de tecnologias de medição padronizadas, como o PISA, e que os dados gerados por essas tecnologias nos dizem coisas importantes sobre a competitividade econômica nacional das nações (LINGARD; SELLAR; SAVAGE, 2014). Em resposta, uma quantidade significativa de pesquisas concentrou-se em criticar essas alegações e examinar os impactos do trabalho da OCDE. A OCDE cria e a pesquisa crítica critica a criação. Embora isso possa parecer lógico, concentrar tanta atenção em uma organização que existe para, em primeiro lugar, gerar atenção pode incorporar ainda mais seu poder global. Este é, sem dúvida, um cenário complicado, pois, embora forças poderosas precisem de crítica, uma atenção maior a essas forças as coloca ainda mais no centro das atenções e pode ampliar sua importância na medida em que outros fatores importantes são ocultados. Nossa preocupação, portanto, não é que a pesquisa seja conduzida sobre a OCDE, mas que tanta atenção levanta uma questão sobre quais outras forças e tecnologias políticas globais estão sendo deixadas de lado como resultado da recente "febre da OCDE" entre sociólogos críticos das políticas educacionais.

Em nossa opinião, um grande desafio teórico e metodológico para a sociologia crítica das políticas é como abordar o que está além da vasta "luminosidade" das redes globais e móveis que comandam nossa atenção, para focar nas contestações políticas e sociais mais amplas em torno das práticas educacionais que não podem tão facilmente ser rastreadas e "seguidas" (GERRARD, 2015). Isso, por sua vez, levanta questões sobre como a própria "mobilidade" é entendida e colocada em ação (enacted) dentro da sociologia crítica das políticas, particularmente dada a contínua centralidade das fronteiras nacionais e subnacionais na constituição de espaços políticos e das políticas educacionais. Na verdade, preocupamo-nos que as formas de sociologia crítica das políticas, centradas, principalmente, na compreensão das formas de mobilidade, corram o risco de ofuscar formas de entorpecimento, imobilidade e lentidão. Proponente de estudos de mobilidade, Urry (2003) abordou essas dinâmicas na dialética conceitual "mobilidade/amarras", que indica o caráter relacional da mobilidade como envolvendo imobilidade infraestrutural, material e social (ver também BISSELL; FULLER, 2013). Tentativas semelhantes de lidar com essa dinâmica existem na pesquisa de mobilidade de políticas que se baseia na teoria do agenciamento para enfatizar o contexto e colocar as formas específicas de como as racionalidades e as tecnologias das políticas se manifestam (PRINCE, 2017; SAVAGE, 2020).

Práxis Educativa, Ponta Grossa, v. 17, e2219696, p. 1-18, 2022

Disponível em: <https://revistas2.uepg.br/index.php/praxiseducativa $>$ 
I/mobilidade, portanto, não é um binário, com forças móveis e imóveis puxando em qualquer direção, mas indica como as mobilidades e as amarras são mutuamente constituídas, dependendo e se capacitando mutuamente. "A vida social”, como Urry (2003, p. 138) argumenta, é "[...] cada vez mais constituída por meio de mundos materiais que envolvem novas e distintas amarras que permitem, produzem e pressupõem novas mobilidades extensas". Um ponto semelhante foi enfatizado por geógrafos críticos, incluindo McCann (2011), que examina a dialética da fixidez/do fluxo, e como fenômenos que exibem maior estabilidade e especificidade de lugar são tão importantes quanto o que é mais fluido/móvel. Como McCann (2011, p. 123) argumenta, com base em Peck e Theodore (2008), “[...] embora o conhecimento possa ser entendido como 'fluindo' ao redor do mundo, ele só é 'acionável' e produtivo quando está incorporado ou territorializado em contextos sociais, espaciais e institucionais específicos”. Como tal, há necessidade de "[...] uma abordagem metodológica sensível tanto ao movimento [...]" quanto "[...] às experiências variáveis de incorporação e transformação em andamento em locais 'a jusantes' de adoção/emulação” (PECK; THEODORE, 2012, p. 24). Robinson (2015) faz uma afirmação semelhante, argumentando que, em vez de "[...] focar no que está se movendo (traçando as trajetórias de um documento de política, uma ideia, um consultor de política)", devemos começar com o local, para "[...] olhar, em vez disso, sobre como os formuladores de políticas compõem suas ideias em meio a uma miríade de influências de outros locais" (ROBINSON, 2015, p. 831). Com base nos argumentos de Robinson, sugerimos que há a necessidade de uma sociologia crítica das políticas educacionais não apenas para abordar a mobilidade em si, mas também as formas como os atores das políticas atraem e combinam várias ideias e práticas de outros locais (muitas vezes em partes fragmentadas) na construção de formulários de políticas específicos do local. $\mathrm{O}$ ponto central, aqui, é como arranjos políticos e das políticas únicos servem para solidificar e cimentar ideias e práticas de maneira que resultem em novas formas que não podem ser simplesmente entendidas como ideias globais tornadas locais (ver KENWAY; FAHEY, 2008).

Relacionado a isso, também levamos em consideração o alerta de Kalir (2013, p. 314) contra a tentação de deixar a "metáfora aquática dos fluxos" ser superestimada à custa de compreender o que permanece ou "gruda". Frequentemente, há uma fetichização do fluxo na pesquisa de sociologia das políticas contemporânea ou, como McCann (2011, p. 109) coloca, uma tendência a "[...] fetichizar as políticas como objetos naturalmente móveis". Conceituar e responder às condições de mobilidade também requer atenção em como a própria mobilidade é configurada pelas necessidades de capital e negócios para tornar algumas coisas e algumas pessoas móveis e outras não. Isso envolve mediar um foco no que é novo, rápido e atraente com a abordagem do passado e das políticas, materialidades e socialidades que são duradouras e sustentáveis (GERRARD; RUDOLPH; SRIPRAKASH, 2017). Novamente, não estamos tentando descrever as formas de entorpecimento como opostas ao movimento. Em vez disso, o dinamismo da prática das políticas envolve necessariamente fixidez $e$ fuga, uma vez que conecta o passado, o presente e o futuro.

Assim, embora concordemos que é cada vez mais impossível entender a política educacional sem uma atenção ao fluxo e à mobilidade, acreditamos ser igualmente importante perguntar: Quais são as imobilidades da política contemporânea? Ao perguntar isso, não estamos sugerindo que pode haver algumas coisas que nunca se movem (ou seja, permanecem imóveis por tempos imemoriais). Em vez disso, nosso argumento é que algumas coisas aderem mais do que outras, tanto temporal quanto espacialmente, e que a aderência é, muitas vezes, parte do que possibilita as condições de mobilidade e "política rápida". As mobilidades, portanto, produzem fortes amarras (o que podemos chamar de "policy mooralities", para cunhar um novo termo, que fala às forças de ancoragem distintas das mobilidades) por meio da produção de racionalidades políticas

${ }^{8}$ Nota de tradução: Os autores reúnem os termos mooring (amarras) e mobilities (mobilidades).

Práxis Educativa, Ponta Grossa, v. 17, e2219696, p. 1-17, 2022 Disponível em: <https://revistas2.uepg.br/index.php/praxiseducativa> 
A política da sociologia crítica das políticas: mobilidades, amarras e redes de elite

e tecnologias semelhantes em vários espaços. E a mobilidade depende dessa moralidade: social, política e materialmente. A pesquisa deve, portanto, manter a atenção tanto no mundo dos fluxos quanto nas práticas relacionadas de fixidez e de entorpecimento.

\section{Criticidade em contextos de mudança}

Décadas depois dos trabalhos de base que moldaram a sociologia crítica das políticas, a necessidade de os pesquisadores pensarem e agirem criticamente permanece tão importante como sempre. $\mathrm{Na}$ verdade, as principais preocupações sociais, políticas e econômicas que orientam o desenvolvimento do campo evoluíram em direções novas e altamente problemáticas que requerem interrogatório crítico contínuo. Por exemplo, o neoliberalismo não está mais "em ascensão", mas agora está consolidado como o principal modo de governança nas economias liberais avançadas, produzindo impactos e desigualdades sociais, econômicas e educacionais que excedem em muito os medos imaginados expressos por pesquisadores críticos nas décadas de 1980 e 1990. Ao entrarmos na década de 2020, enfrentamos uma emergência climática global que supera muitas das previsões mais sombrias das décadas anteriores e, fundamentalmente, ameaça a vida em nosso planeta. As contradições racializadas da "globalização", caracterizadas pela intensificação dos controles de fronteira e a profunda imobilidade (e encarceramento) de muitos, também representam sérios desafios para a governança, a sociedade e a humanidade (GERRARD, 2017). $\mathrm{E}$, apesar do aumento do acesso dos alunos aos níveis superiores, os sistemas educacionais tornaram-se muito mais geradores de desigualdades, com o poder global concentrado nas mãos daqueles que frequentaram as escolas e as universidades de elite do mundo (GALE; MOLLA; PARKER, 2017). Contornando poderosamente todas essas dinâmicas, está a pandemia da Covid19, que vem testando os fundamentos econômicos, políticos e sociais das nações em todo o mundo. No momento da escrita deste texto, uma proporção significativa de alunos nem mesmo frequenta instituições de ensino e muitos estão aprendendo de forma online. A pandemia tem grande potencial para aprofundar ainda mais as desigualdades e remodelar os próprios significados e práticas da educação.

$\mathrm{Na}$ ausência de pesquisas e ações críticas em andamento, essas questões, e muitas outras, têm o potencial de produzir resultados catastróficos nos anos e nas décadas que virão. O papel da pesquisa educacional em contribuir para a produção de conhecimento e estratégias, para navegar em nosso caminho coletivo para frente, dificilmente pode ser subestimado. Pensar sobre como a educação se relaciona com o futuro, à luz dessas preocupações, levanta sérias questões sobre as responsabilidades que "nós", como pesquisadores críticos, temos em relação à compreensão e à reimaginação do passado, do presente e do futuro. Embora a necessidade de criticidade permaneça crucial, o que significa envolver-se em "sociologia crítica das políticas", é improvável que permaneça o mesmo, e o olhar político dos pesquisadores precisará mudar e adaptar-se às mudanças nos contextos empíricos, teóricos e metodológicos. Quando a sociologia crítica das políticas emergiu na década de 1980, os estudiosos estavam reagindo e imaginando como deveria ser um projeto crítico das políticas que abordasse as questões predominantes da época. Enquanto os elementos centrais dessas articulações permanecem relevantes em "novos tempos", agora somos apresentados a novos fenômenos a serem pesquisados e compreendidos criticamente, novas formas de pensar sobre o mundo, novas formas de conduzir pesquisas e novas condições de possibilidade para o trabalho acadêmico.

O objetivo principal deste artigo é agitar o campo, levantar questões sobre o que significa ser um "sociólogo crítico das políticas" na mudança de contextos teóricos, empíricos e metodológicos na educação. Nosso objetivo não foi mapear de forma abrangente o campo e suas preocupações, mas adotar uma abordagem direcionada com foco em duas considerações primárias.

Práxis Educativa, Ponta Grossa, v. 17, e2219696, p. 1-18, 2022 Disponível em: <https://revistas2.uepg.br/index.php/praxiseducativa $>$ 
Em primeiro lugar, refletimos sobre a política da criticidade, considerando diferentes afirmações e debates sobre o que significa engajar-se na pesquisa crítica e sociológica da política educacional. Prestamos atenção especial aos posicionamentos políticos implícitos e explícitos dos pesquisadores e como os pesquisadores posicionam seu trabalho em relação ao poder da elite e às redes de políticas. Em segundo lugar, contamos com essas bases para considerar o rápido aumento da pesquisa de mobilidades, com um foco particular na abordagem popular de "seguir a política". Argumentamos que, embora esta pesquisa fale de uma necessidade crucial de examinar o poder e o impacto das redes globais de elite, ela também corre o risco de orientar os pesquisadores para problemas e agendas já estabelecidas por agentes políticos de elite e de organizações, enquanto obscurece as forças não tão móveis (isto é, mooralities) que não servem apenas como forças de ancoragem, mas fornecem infraestrutura para permitir fluxos móveis. O desejo de seguir os fluxos de políticas globais também levanta questões sobre as redes de elite e os níveis privilegiados de recursos necessários para conduzir tais pesquisas. Vemos um grande perigo na pesquisa que conta com o elitismo como uma pré-condição para o engajamento, questionando se os pesquisadores que buscam "criticar" as redes de elite podem, em vez disso, estar participando da pesquisa das elites, pelas elites e para as elites.

Olhando para o futuro, vemos os argumentos neste artigo como levantando questões sobre a política de produção de conhecimento e destacando novos desafios para sociólogos das políticas que buscam "ser críticos" em contextos de mudança. Sugerimos que haja uma necessidade maior de criticar o crítico, mas enfatizamos que isso também deve envolver uma crítica autorreflexiva contínua de nossas próprias reivindicações de criticidade, bem como atenção crítica às áreas empíricas de enfoque que escolhemos examinar (ou alternativamente, são ignorados ou obscurecidos de nossa visão). Como argumentamos, a política de reivindicar ser um intelectual crítico está ligada à posição de nossa pesquisa nas realidades sociais, políticas e econômicas que buscamos criticar. Assim sendo, embora acreditemos que haja um escopo significativo para "agir" no mundo, por meio da pesquisa, e esperemos que tais esforços possam levar a uma mudança positiva, qualquer ação deve ser entendida como constituída dentro das condições que a sustentam e a produzem. Manter uma postura crítica autorreflexiva não apenas em relação aos interesses de pesquisa, mas também em relação ao que significa ser um pesquisador crítico, é, portanto, central para tornar-se, ser e evoluir como um sociólogo crítico das políticas.

\section{Declaração de divulgação}

Nenhum conflito de interesse foi relatado pelos autores.

\section{Informações adicionais}

Financiamento

Este trabalho recebeu apoio do Australian Research Council [DP190101867].

\section{Sobre os autores}

Glenn C. Savage é um sociólogo das políticas com experiência em reforma educacional, federalismo, relações intergovernamentais e mobilidades políticas globais. Ele é professor associado da University of Western Australia na School of Social Sciences e na Graduate School of Education.

Jessica Gerrard pesquisa as formações em mudança e experiências vividas de desigualdades sociais em relação à educação, ao ativismo, ao trabalho e ao desemprego. Ela trabalha nas disciplinas de Sociologia, História e Estudos de Políticas, com interesse em metodologias e teorias críticas. 
Trevor Gale é catedrático de Política Educacional e Justiça Social na University of Glasgow. Ele é um sociólogo crítico da educação com interesses de pesquisa na reprodução das desigualdades em e por meio de políticas e práticas em sistemas de educação formais, particularmente em escolas e no Ensino Superior.

Tebeje Molla é membro do Discovery Early Career Researcher (DECRA) na Escola de Educação da Deakin University, Austrália. Sua pesquisa concentra-se na desigualdade educacional e nas respostas das políticas nos níveis sistêmicos e institucionais. Teoricamente, seu trabalho é orientado pela sociologia crítica e pela abordagem da capacidade para a justiça social e o desenvolvimento humano.

Agradecimento: Este artigo, originalmente intitulado The politics of critical policy sociology: mobilities, moorings and elite networks, de Glenn C. Savage, Jessica Gerrard, Trevor Gale e Tebeje Molla, copyright (C) 2021, foi publicado em Critical Studies in Education 2021, v. 62, n. 3, copyright (C) Taylor \& Francis Ltd. 2021, reimpresso com permissão do editor.

Acknowledgement: "The politics of critical policy sociology: mobilities, moorings and elite networks" by Glenn C. Savage, Jessica Gerrard, Trevor Gale \& Tebeje Molla copyright (C) 2021 Critical Studies in Education 2021, Vol. 62 issue 3, copyright (CTaylor \& Francis Ltd 2021, reprinted by permission of The publisher.

\section{Referências}

APPLE, M. W. Power, meaning and identity: Critical sociology of education in the United States. British Journal of Sociology of Education, v. 17, n. 2, p. 125-144, 1996. DOI: https://doi.org/10.1080/0142569960170201

BACCHI, C. Policy as discourse: What does it mean? Where does it get us? Discourse: Studies in the Cultural Politics of Education, v. 21, n. 1, p. 45-57, 2000. DOI: https://doi.org/10.1080/01596300050005493

BALL, S. J. Policy sociology and critical social research: A personal review of recent education policy and policy research. British Educational Research Journal, v. 23, n. 3, p. 257-274, 1997. DOI: https://doi.org/10.1080/0141192970230302

BALL, S. J. Following policy: Networks, network ethnography and education policy mobilities. Journal of Education Policy, v. 31, n. 5, p. 549-566, 2016. DOI: https://doi.org/10.1080/02680939.2015.1122232

BECK, U. The cosmopolitan condition: Why methodological nationalism fails. Theory, Culture $\boldsymbol{\&}$ Society, v. 24, n. $7-8, \quad$ p. 286-290, 2007. DOI: https://doi.org/10.1177/02632764070240072505

BERLANT, L. Cruel optimism. Durham: Duke University Press, 2011.

BISSELL, D.; FULLER, G. (ed.). Stillness in a mobile world. London: Routledge, 2013.

BLACKMORE, J. Cultural and gender politics in Australian education, the rise of edu-capitalism and the 'fragile project' of critical educational research. Australian Educational Researcher, v. 41, n. 5, p. 499-520, 2014. DOI: https://doi.org/10.1007/s13384-014-0158-8

Práxis Educativa, Ponta Grossa, v. 17, e2219696, p. 1-18, 2022 Disponível em: <https://revistas2.uepg.br/index.php/praxiseducativa> 
BOURDIEU, P.; WACQUANT, L. J. D. An invitation to reflexive sociology. Chicago: University of Chicago Press, 1992.

CASTELLS, M. The rise of the network society. Oxford: Blackwell, 1996.

DAVID, M.; WEINER, G.; ARNOT, M. Gender equality and schooling, education policy-making and feminist research in England and Wales in the 1990s. In: SALISBURY, J.; RIDDELL, S. (ed.). Gender, policy, and educational change: Shifting agendas in the UK and Europe. London: Routledge, 2000. p. 19-36.

DUKE, K. Getting beyond the 'official line': Reflections on dilemmas of access, knowledge and power in researching policy networks. Journal of Social Policy, v. 31, n. 1, p. 39-59, 2002. DOI: https://doi.org/10.1017/S0047279402006505

FENWICK, T.; EDWARDS, R. Actor-network theory in education. London: Routledge, 2010.

GALE, T. Critical policy sociology: Historiography, archaeology and genealogy as methods of policy analysis. Journal of Education Policy, v. 16, n. 5, p. 379-393, 2001. DOI: https://doi.org/10.1080/02680930110071002

GALE, T. Realizing policy: The who and how of policy production. Discourse: Studies in the Cultural Politics of Education, v. 24, n. 1, p. 51-66, 2003. DOI: https://doi.org/10.1080/01596300303026

GALE, T.; MOLLA, T.; PARKER, S. The illusion of meritocracy and the audacity of elitism: Expanding the evaluative space in education. In: PARKER, S.; GULSON, K. N.; GALE, T. (ed.). Policy and inequality in education. London: Springer, 2017. p. 7-21.

GERRARD, J. Counterpublics, crisis and critique: A feminist socio-historical approach to researching policy. In: GULSON, K.; CLARKE, M.; BENDIX-PETERSON, E. (ed.). Education policy and contemporary theory: Implications for research. London: Routledge, 2015. p. 122133.

GERRARD, J. The refugee crisis, non-citizens, border politics and education. Discourse: Studies in the Cultural Politics of Education, v. 38, n. 6, p. 880-891, 2017. DOI: https://doi.org/10.1080/01596306.2016.1227959

GERRARD, J.; RUDOLPH, S.; SRIPRAKASH, A. The politics of post-qualitative inquiry: History and power. Qualitative Inquiry, v. 23, n. 3, p. 384-394, 2017. DOI: https://doi.org/10.1177/1077800416672694

GILLBORN, D. Education policy as an act of white supremacy: Whiteness, critical race theory and education reform. Journal of Education Policy, v. 20, n. 4, p. 485-505, 2005. DOI: https://doi.org/10.1080/02680930500132346

GORUR, R. et al. Politics by other means? STS and research in education. Discourse: Studies in the Cultural Politics of Education, v. 40, n. 1, p. 1-15, 2019. DOI: https://doi.org/10.1080/01596306.2018.1549700

GULSON, K. N. et al. Policy mobilities and methodology: A proposition for inventive methods in education policy studies. Critical Studies in Education, v. 58, n. 2, p. 224-241, 2017. DOI: https://doi.org/10.1080/17508487.2017.1288150 
GUNTER, H. The politics of public education: Reform ideas and issues. Bristol: Policy Press, 2018.

HARTONG, S.; PIATTOEVA, N. Contextualizing the datafication of schooling: A comparative discussion of Germany and Russia. Critical Studies in Education, p. 1-16, 2019. DOI: https://doi.org/10.1080/17508487.2019.1618887

HOGAN, A. NAPLAN and the role of edu-business: New governance, new privatisations and new partnerships in Australian education policy. The Australian Educational Researcher, v. 43, n. 1, p. 93-110, 2016. DOI: https://doi.org/10.1007/s13384-014-0162-z

HOGARTH, M. Talkin' bout a revolution: The call for transformation and reform in indigenous education. The Australian Educational Researcher, v. 45, n. 5, p. 663-674, 2018. DOI: https://doi.org/10.1007/s13384-018-0277-8

HORKHEIMER, M. Critical theory. New York: Seabury Press, 1972.

KALIR, B. Moving subjects, stagnant paradigms: Can the 'mobilities paradigm' transcend methodological nationalism? Journal of Ethnic and Migration Studies, v. 39, n. 2, p. 311-327, 2013. DOI: https://doi.org/10.1080/1369183X.2013.723260

KENWAY, J.; FAHEY, J. Imagining research otherwise. In: KENWAY, J.; FAHEY, J. (ed.). Globalizing the Research Imagination. London: Routledge, 2008. p. 1-40.

KENWAY, J.; MCLEOD, J. Bourdieu's reflexive sociology and 'spaces of points of view': Whose reflexivity, which perspective? British Journal of Sociology of Education, v. 25, n. 4, p. 525-544, 2004. DOI: https://doi.org/10.1080/0142569042000236998

LATOUR, B. Politics of nature: How to bring the sciences into democracy. Cambridge: Harvard University Press, 2004.

LEWIS, S. The turn towards policy mobilities and the theoretical-methodological implications for policy sociology. Critical Studies in Education, p. 1-16, 2020. DOI: https://doi.org/10.1080/17508487.2020.1808499

LEWIS, S.; HOGAN, A. Reform first and ask questions later? The implications of (fast) schooling policy and 'silver bullet' solutions. Critical Studies in Education, v. 60, n. 1, p. 1-18, 2019. https://doi.org/10.1080/17508487.2016.1219961

LINGARD, B. The changing state of policy production in education: Some Australian reflections on the state of policy sociology. International Studies in Sociology of Education, v. 3, n. 1, p. 25-47, 1993. DOI: https://doi.org/10.1080/0962021930030102

LINGARD, B.; SELLAR, S.; SAVAGE, G. C. Re-articulating social justice as equity in schooling policy: The effects of testing and data infrastructures. British Journal of Sociology of Education, v. 35, n. 5, p. 710-730, 2014. DOI: https://doi.org/10.1080/01425692.2014.919846

MAYLOR, U. Is it because I'm Black? A Black female research experience. Race Ethnicity and Education, v. 12, n. 1, p. 53-64, 2009. DOI: https://doi.org/10.1080/13613320802650949

MCCANN, E. Urban policy mobilities and global circuits of knowledge: Toward a research agenda. Annals of the Association of American Geographers, v. 101, n. 1, p. 107-130, 2011. DOI: https://doi.org/10.1080/00045608.2010.520219 
METCALFE, A. S. Nomadic political ontology and transnational academic mobility. Critical Studies in Education, v. 58, p. 2, p. 131-149, 2017. DOI: https://doi.org/10.1080/17508487.2016.1264987

MILLS, C. The sociological imagination. Oxford: Oxford University Press, 1959.

MILNER, M. Elites: A general model. Oxford: Polity, 2015.

MIRZA, H. The in/visible journey: Black women's lifelong lessons in higher education. In: LEATHWOOD, C.; FRANCIS, B. (ed.). Gender and lifelong learning: Critical feminist engagements. London: Routledge, 2006. p. 137-152.

NEAL, S.; MCLAUGHLIN, E. Researching up? Interviews, emotionality and policy-making elites. Journal of Social Policy, v. 38, n. 4, p. 689-707, 2009. DOI: https://doi.org/10.1017/S0047279409990018

ONG, A. Neoliberalism as a mobile technology. Transactions of the Institute for British Geographers, v. 32, n. 1, p. 3-8, 2007.

OZGA, J. Researching the powerful: Seeking knowledge about policy. European Educational Research Journal, v. 10, n. 2, p. 218-224, 2011. DOI: https://doi.org/10.2304/eeri.2011.10.2.218

OZGA, J. Problematising policy: The development of (critical) policy sociology. Critical Studies in Education, v. 62, n. 3, 2019, p. 290-305. DOI: https://doi.org/10.1080/17508487.2019.1697718

OZGA, J.; GEWIRTZ, S. Sex, lies and audiotape: Interviewing the education policy elite. In: HALPIN, D.; TROYNA, B. (ed.). Researching education policy: Ethical and methodological issues. Basingstoke: Falmer Press, 1994. p. 145-167

PECK, J.; THEODORE, N. Embedding policy mobilities. Working paper, Department of Geography, Vancouver: University of British Columbia, 2008.

PECK, J.; THEODORE, N. Follow the policy: A distended case approach. Environment and Planning A, v. 44, n. 1, p. 21-30, 2012. DOI: https://doi.org/10.1068/a44179

PECK, J.; THEODORE, N. Fast Policy. Minnesota: University of Minnesota Press, 2015.

PRINCE, R. Local or global policy? Thinking about policy mobility with assemblage and topology. Area, v. 49, n. 3, p. 335-341, 2017. DOI: https://doi.org/10.1111/area.12319

PRUNTY, J. Signposts for a critical educational policy analysis. Australian Journal of Education, v. 29, n. 2, p. 133-140, 1985. DOI: https://doi.org/10.1177/000494418502900205

RASMUSSEN, M. 'Cruel Optimism' and contemporary Australian critical theory in educational research. Educational Philosophy and Theory, v. 47, n. 2, p. 192-206, 2016. DOI: https://doi.org/10.1080/00131857.2013.793929

REGMI, K. Critical policy sociology: Key underlying assumptions and their implications for educational policy research. International Journal of Research \& Method in Education, v. 42, n. 1, p. 59-75, 2019. DOI: https://doi.org/10.1080/1743727X.2017.1398228

RIZVI, F.; LINGARD, B. Globalizing education policy. London: Routledge, 2010. 
ROBINSON, J. 'Arriving At' urban policies: The topological spaces of urban policy mobility. International Journal of Urban and Regional Research, v. 39, n. 4, p. 831-834, 2015. DOI: https://doi.org/10.1111/1468-2427.12255

SAVAGE, M. Introduction to elites: From 'the problematic of the proletariat' to a class analysis of 'wealth elites'. The Sociological Review, v. 63, n. 2, p. 223-239, 2015. DOI: https://doi.org/10.1111/1467-954X.12281

SAVAGE, G. C. Think tanks, education and elite policy actors. The Australian Educational Researcher, v. 43, n. 1, p. 35-53, 2016. DOI: https://doi.org/10.1007/s13384-015-0185-0

SAVAGE, G. C. What is policy assemblage? Territory, Politics, Governance, v. 8, n. 3, p. 319335, 2020. DOI: https://doi.org/10.1080/21622671.2018.1559760

SAVAGE, M.; WILLIAMS, K. Elites: remembered in capitalism and forgotten by social sciences. The Sociological Review, v. 56, n. 1, p. 1-24, 2008. DOI: https://doi.org/10.1111/j.1467954X.2008.00759.x

SCOTT, J. Modes of power and the re-conceptualisation of elites. The Sociological Review, v. 56, n. 1, p. 25-43, 2008. DOI: https://doi.org/10.1111/j.1467-954X.2008.00760.x

SCOTT, J. P. Critical social theory: An introduction and critique. The British Journal of Sociology, v. 29, n. 1, p. 1-21, 1978. DOI: https://doi.org/10.2307/589216

SELLAR, S.; SAVAGE, G. C.; GORUR, R. The politics of disagreement in critical education policy studies: A response to Morsy, Gulson and Clarke. Discourse: Studies in the Cultural Politics of Education, v. 35, n. 3, p. 462-469, 2014. DOI: https://doi.org/10.1080/01596306.2014.890269

SMITH, L. T. Decolonizing methodologies: research and indigenous peoples. London: Zed Books, 1999.

THOMPSON, G. Learning personalisation: Technics, disorientation and governance. In: WILKINS, A.; OLMEDO, A. (ed.). Education governance and social theory: Interdisciplinary approaches to research. London: Bloomsbury Academic, 2019. p. 43-62.

THUNIG, A.; JONES, T. 'Don't make me play house-n***er: Indigenous academic women treated as 'black performer' within higher education. The Australian Educational Researcher, 48, p. 397-417, 2020. DOI: https://doi.org/10.1007/s13384-020-00405-9

URRY, J. Global complexity. Oxford: Polity Press, 2003.

YOUNG, M, F. D (ed.). Knowledge and control: new directions for the sociology of education. London: Collier-Macmillan Publishers, 1971.

Recebido em 15/02/2021

Permissão para tradução recebida em 28/09/2021

Publicado online em 19/10/2021

Práxis Educativa, Ponta Grossa, v. 17, e2219696, p. 1-18, 2022 Disponível em: <https://revistas2.uepg.br/index.php/praxiseducativa $>$ 